\title{
Advancing Inverse Sensitivity/Uncertainty Methods for Nuclear Fuel Cycle Applications
}

\author{
G. Arbanas, ${ }^{1, *}$ M.L. Williams, ${ }^{1}$ L.C. Leal,${ }^{1}$ M.E. Dunn, ${ }^{1}$ B.A. Khuwaileh, ${ }^{2}$ C. Wang, ${ }^{2}$ and H. Abdel-Khalik ${ }^{2}$ \\ ${ }^{1}$ Reactor and Nuclear Systems Division, Oak Ridge National Laboratory, Oak Ridge, TN 37831-6171, USA \\ ${ }^{2}$ Department of Nuclear Engineering, North Carolina State University, Raleigh, NC 27695-7909, USA
}

(Received 29 May 2014; revised received 15 September 2014; accepted 1 October 2014)

\begin{abstract}
The inverse sensitivity/uncertainty quantification (IS/UQ) method has recently been implemented in the Inverse Sensitivity/UnceRtainty Estimator (INSURE) module of the AMPX cross section processing system [1]. The IS/UQ method aims to quantify and prioritize the cross section measurements along with uncertainties needed to yield a given nuclear application(s) target response uncertainty, and doing this at a minimum cost. Since in some cases the extant uncertainties of the differential cross section data are already near the limits of the present-day state-of-the-art measurements, requiring significantly smaller uncertainties may be unrealistic. Therefore, we have incorporated integral benchmark experiments (IBEs) data into the IS/UQ method using the generalized linear least-squares method, and have implemented it in the INSURE module. We show how the IS/UQ method could be applied to systematic and statistical uncertainties in a self-consistent way and how it could be used to optimize uncertainties of IBEs and differential cross section data simultaneously. We itemize contributions to the cost of differential data measurements needed to define a realistic cost function.
\end{abstract}

\section{INTRODUCTION}

Improving the quality of evaluated nuclear differential cross section data and the IBEs is expected to enable more accurate modeling of nuclear applications [2]4. Costs incurred by measurement of nuclear cross sections include the operating cost of experimental facilities, their scientific and technical support personnel, neutron target preparation, detectors, data reduction, and data evaluation, which can lead to a combined cost on the order of $\$ 400,000 /$ measurement [5. Measurements of IBEs can cost hundreds of thousands of dollars or more depending on the complexity and difficulty of the experiment.

Because of such high costs, it is advantageous to optimize nuclear data efforts by prioritizing nuclear data measurements in the most efficient sequence that achieves the application's target response accuracy. This is the purpose of the Inverse Sensitivity/Uncertainty Quantification (IS/UQ) methods originally initiated by Uscachev [6]. One of the early implementations of the

\footnotetext{
* Corresponding author: arbanasg@ornl.gov

This manuscript has been authored by UT-Battelle LLC under Contract No. DE-AC05-00OR22725 with the US Department of Energy. The US government retains and the publisher, by accepting the article for publication, acknowledges that the US government retains a nonexclusive, paid-up, irrevocable, worldwide license to publish or reproduce the published form of this manuscript, or allow others to do so, for US government purposes.
}

IS/UQ method was in the ORNL's legacy computer codes FORSS and NUTCRACKER [7, which were applied to fast reactor analysis previously. Subsequent implementations of the IS/UQ method that preceded our work have been reported in 8 11] and were targeted to applications that are sensitive mostly to cross sections above $50 \mathrm{keV}$.

In our previous work, we applied IS/UQ to thermal and medium energy systems, where it is known that selfshielded cross sections and sensitivities should be used, and we quantified the effect of self-shielding. [12. Also, we recently incorporated IBEs in the IS/UQ method and suggested that they could relieve demands on differential data uncertainty [13. We have done this by employing standard data and covariance adjustment techniques for IBEs that have been encoded in the TSURFER module [14, 15] of the SCALE system [4, 16]. This technique will be helpful especially for cases where extant cross section uncertainties are already measured as precisely as possible in today's state-of-the art experiments.

In Sec. II we review the extant IS/UQ method formalism that has been implemented in the new IS/UQ code INSURE [17] that will become part of the AMPX cross-section processing system [1] under a modern SQA framework. In Sec. III we show how the IS/UQ method could be adapted to simultaneously optimize systematic and statistical sources of uncertainty. It is hoped that by phrasing the IS/UQ problem in terms of systematic and statistical sources of uncertainty, the IS/UQ method would provide more intuitive guidelines for the experimentalist for reducing the overall uncertainties. In Sec. 
IV we show how the IS/UQ could be extended to optimize the uncertainties of the IBEs simultaneously with the uncertainties in the differential data. In Sec. V we discuss contributions to the cost function, and in Sec. VI we show a simple analytically solvable IS/UQ example.

\section{INVERSE SENSITIVITY/UNCERTAINTY QUANTIFICATION (IS/UQ) METHOD}

In this section the IS/UQ method and our prior work are summarized. We define a cost function that is to be minimized by the IS/UQ method, and constraints that are to be satisfied. All formal expressions are to the firstorder perturbation approximation that we assume to be reasonably accurate on the scale of uncertainties in nuclear data. This first-order expansion is also consistent with the approximation that is used in the SCALE module TSURFER for adjustment of the nuclear data uncertainties. The formalism is first presented without using IBEs, but these are incorporated later.

We seek to minimize the cost of an improved uncertainties contained in the improved covariance matrix labeled $\mathbf{C}^{\prime}$ to distinguish it from the extant covariance matrix $\mathbf{C}$, where the cost can be defined as the sum of inverse of the diagonal elements of $\mathbf{C}^{\prime}$

$$
\min \left[\operatorname{cost}\left[\mathbf{C}^{\prime}\right]\right]=\min \left[\sum_{i} \frac{\lambda_{i}}{C_{i i}^{\prime}}\right],
$$

where $\lambda_{i}$ 's are cost weights that reflect varying cost of materials, cross sections, etc. A composite index $i$ consists of the energy-group index, material index, and the cross section kind index. This simple cost definition is equivalent to those used in the past [9]. For the applications described in this work, the covariance matrix $\mathbf{C}$ is the SCALE's 44-group relative covariance matrix, which is also conventionally used by the TSURFER module. This cost minimization is constrained by maximum allowed tolerances $\delta_{\mathbf{R}}$ of application's responses $\mathbf{R}$

$$
\operatorname{diag}\left[\mathbf{S C}^{\prime} \mathbf{S}^{T}\right] \leq \operatorname{var}[\mathbf{R}],
$$

where $\mathbf{S}$ is a (relative) sensitivity matrix of response vector $\mathbf{R}$ w.r.t. to a group-wise differential cross section $\sigma_{i}$

$$
S_{j i} \equiv \frac{\sigma_{i}}{R_{j}} \frac{\partial R_{j}}{\partial \sigma_{i}},
$$

which is computed by the TSUNAMI module of the SCALE system, which takes into account cross section self-shielding [12]. The IS/UQ problem is conventionally defined by parameterizing the covariance matrix $\mathbf{C}^{\prime}$ via variables $x_{i}$ that relate its elements to those of the extant covariance matrix $\mathbf{C}$

$$
C_{i j}^{\prime} \equiv x_{i} C_{i j} x_{j}
$$

The $x_{i}$ 's are coefficients that are varied until all constraints are satisfied at a minimum cost. This parameter- ization preserves the correlation of the extant covariance matrix C. (An alternative and novel parameterization that allows simultaneous optimization of systematic and statistical uncertainties is presented in Sec. III.) We observe that $x_{i}$ could be restricted to be less than or equal to 1 because any value larger than 1 would correspond to data uncertainties that are greater than those already present in $\mathbf{C}$, and the cost of $x_{i}=1$ can be set to zero because it corresponds to the uncertainty of the extant data. In principle, one could set the lower bound for $x_{i}$ by allowing the diagonal elements of the covariance $C_{i i}^{\prime}=x_{i}^{2} C_{i i}$ to be no smaller than the smallest uncertainties presently achievable for various cross sections, as prescribed in the ENDF [3], such as $1 \%$ for total cross section, $2 \%$ for capture cross section, etc. However, we have found that imposing these lower limits on the uncertainties (especially on the data to which the application is most sensitive to) makes it impossible for the constraint on the application's target response to be satisfied. Therefore we have released this constraint on the lower limit of $x_{i}$, but we mitigate unrealistically small $x_{i}$ by incorporating IBEs data.

The effect of IBEs on an application is taken into account via the Generalized Linear Least-squares (GLLS) formalism using a first-order expansion. In this work we follow the template implementation in the SCALE module TSURFER 14, 15]. We state the relevant expressions for a reduction (or adjustment) of a covariance matrix $\mathbf{C}$ due to IBEs, applied to covariance matrix $\mathbf{C}^{\prime}$ that is being optimized

$$
\mathbf{C}^{\prime \prime}=\mathbf{C}^{\prime}-\mathbf{C}^{\prime} \mathbf{S}^{T}\left(\mathbf{S C}^{\prime} \mathbf{S}^{T}+\mathbf{F}_{\frac{m}{k}} \mathbf{C}_{\mathbf{m m}} \mathbf{F}_{\frac{m}{k}}\right)^{-1} \mathbf{S C}^{\prime},
$$

where $\mathbf{C}_{\mathbf{m m}}$ is a covariance matrix of responses of IBEs and applications, $\mathbf{S}$ is a sensitivity matrix of their responses with respect to nuclear data, and $\mathbf{F}_{\frac{m}{k}}$ is a diagonal matrix of ratios of experimental to calculated response values. This ratio is set to 1 for applications since (by definition) their responses have not been measured. Inserting this adjusted expression for $\mathbf{C}^{\prime}$, given by Eq. (5), into the expression for the constraint in Eq. (2) yields

$$
\operatorname{diag}\left[\mathbf{S C}^{\prime \prime} \mathbf{S}^{T}\right] \leq \operatorname{var}[\mathbf{R}] .
$$

This expression suggests that this constraint can be satisfied with larger uncertainties in the differential data due to the reduction of the covariance matrix $\mathbf{C}^{\prime}$ by the last term in Eq. (5), as has been demonstrated in [13.

A method for selecting IBEs relevant to an application of interest is based on a similarity of sensitivity profile of an IBE to that of the application; this method has been described in 16.

\section{SYSTEMATIC AND STATISTICAL UNCERTAINTIES}

The IS/UQ method described above does not distinguish between statistical and systematic contributions to 
the overall uncertainties encoded in the original covariance matrix C. In its current form, the IS/UQ optimizes the entire covariance matrix, $\mathbf{C}^{\prime}$, which, once optimized, does not provide insight as to which source of uncertainty the experimentalist ought to try to reduce in a most cost-effective way. Therefore we show below how the IS/UQ method could be adapted to optimize statistical and systematic uncertainties simultaneously, leading to optimized uncertainties that are more meaningful to experimentalists.

Instead of using the covariance data of differential cross section data as the starting point of the IS/UQ method, we separate it formally into systematic and statistical contributions. In this way, the IS/UQ method can provide clearer guidance to the experimentalist as to what sources of uncertainties ought to be reduced in order to achieve the target response uncertainty at a minimum cost. We use the notation of the Sec. III.E.3.b of Ref. 2, where $d$ represent the raw (unreduced) data and $D$ represents the reduced data, which are related via

$$
D_{i}=f_{i}(\{d\},\{q\}),
$$

where $f_{i}$ is some function of the entire set of raw data, $\{d\}$, and of the set of data-reduction parameters, $\{q\}$. The first-order variation of the reduced data $\delta D_{i}$ is then

$$
\delta D_{i}=\sum_{j} \frac{\partial f_{i}}{\partial d_{j}} \delta d_{j}+\sum_{k} \frac{\partial f_{i}}{\partial q_{k}} \delta q_{k} .
$$

Assuming there are no correlations between any two raw data points, that is, $\left\langle\delta d_{j} \delta d_{j^{\prime}}\right\rangle=\Delta_{j}^{2} \delta_{j j^{\prime}}$ and that there are no correlations between raw data and data reduction parameters, that is, $\left\langle\delta q_{k} \delta d_{j}\right\rangle=0$, then the covariance matrix of the reduced data, $C_{i i^{\prime}} \equiv\left\langle\delta D_{i} \delta D_{i^{\prime}}\right\rangle$, can be written as

$$
C_{i i^{\prime}}=\sum_{j} \frac{\partial f_{i}}{\partial d_{j}} \Delta_{j}^{2} \frac{\partial f_{i^{\prime}}}{\partial d_{j}}+\sum_{k k^{\prime}} \frac{\partial f_{i}}{\partial q_{k}} m_{k k^{\prime}} \frac{\partial f_{i^{\prime}}}{\partial q_{k^{\prime}}},
$$

where $m_{k k^{\prime}} \equiv\left\langle\delta q_{k} \delta q_{k^{\prime}}\right\rangle$ is the covariance matrix of the data reduction parameters. In order to optimize systematic and statistical uncertainties in the IS/UQ method, we parametrize the covariance matrix by parameter sets $\{\xi\}$ and $\{\zeta\}$ as

$$
C_{i i^{\prime}}^{\prime} \equiv \sum_{j} \frac{\partial f_{i}}{\partial d_{j}} \Delta_{j}^{\prime 2} \frac{\partial f_{i^{\prime}}}{\partial d_{j}}+\sum_{k k^{\prime}} \frac{\partial f_{i}}{\partial q_{k}} m_{k k^{\prime}}^{\prime} \frac{\partial f_{i^{\prime}}}{\partial q_{k^{\prime}}}
$$

where $\Delta_{j}^{\prime 2} \equiv \xi_{j}^{2} \Delta_{j}^{2}$ and $m_{k k^{\prime}}^{\prime} \equiv \zeta_{k} m_{k k^{\prime}} \zeta_{k^{\prime}}$. The parameter sets $\{\xi\}$ and $\{\zeta\}$ are to be optimized by the IS/UQ, analogously to the parameter set $\{x\}$ in Eq. (4). Although the number of combined parameters in sets $\{\xi\}$ and $\{\zeta\}$ to be optimized may be greater than the number of parameters in set $\{x\}$, the optimization can proceed in a completely analogous way.

The cost function for this parameterization could retain similar to Eq. (1) with $C_{i i}^{\prime}$ taken from Eq. (10). Alternatively, the expression for the cost given in Eq. (1) could be generalized as a sum of costs of statistical and systematic components, each of which has the form similar to that in Eq. (1)

$$
\operatorname{cost}\left[\mathbf{C}^{\prime}\right]=\sum_{j} \frac{\lambda_{\xi_{j}}}{\xi_{j}^{2} \Delta_{j}^{2}}+\sum_{k} \frac{\lambda_{\zeta_{k}}}{\zeta_{k}^{2} m_{k k}} .
$$

The parameterization in Eq. (10), unlike the one in Eq. (4), does not assume that the differential data correlation matrix remains unchanged by the IS/UQ optimization. Perhaps this can be viewed as an advantage of the proposed parameterization, as it would not require the correlation of the original data to be preserved through the IS/UQ optimization.

\section{IS/UQ METHOD FOR INTEGRAL BENCHMARK EXPERIMENTS (IBE)}

In this section we show how the IS/UQ method could be adapted to simultaneously and self-consistently optimize the uncertainties of the differential data and of the IBEs data. To do this, we adopt the efficient notation from the manual of the TSURFER module of the SCALE system, where $\mathbf{C}_{\boldsymbol{\alpha} \boldsymbol{\alpha}}$ is the covariance matrix of the differential data, $\mathbf{C}_{\mathbf{m m}}$ is a block-diagonal matrix of the covariance matrix of measured responses of the IBEs $\left(\mathbf{C}_{\mathbf{b b}}\right)$ and the covariance matrix of the responses of applications $\left(\mathbf{C}_{\mathbf{a a}}\right)$

$$
\mathbf{C}_{\mathbf{m m}} \equiv\left[\begin{array}{cc}
\mathbf{C}_{\mathbf{b b}} & 0 \\
0 & \mathbf{C}_{\mathbf{a a}}
\end{array}\right] \text {. }
$$

Since the applications' responses have not been measured, their uncertainties are (in principle) to be set to infinity. In practice, the TSURFER module of the SCALE system sets their variances, which are diagonal elements of $\mathbf{C}_{\mathbf{a a}}$, to $10^{10}$ as an approximation of infinity.

While the conventional IS/UQ method is focused on optimizing the covariance matrix of the differential data only, that is $\mathbf{C}_{\boldsymbol{\alpha} \boldsymbol{\alpha}}$, the same method could be extended to include $\mathbf{C}_{\mathbf{b b}}$, so that both $\mathbf{C}_{\boldsymbol{\alpha} \boldsymbol{\alpha}}$ and $\mathbf{C}_{\mathbf{b b}}$ and are optimized simultaneously.

For this to take place, the cost of the improved data uncertainties will, in addition to the original cost of the differential data, have an additional component related to the cost of improved IBEs uncertainties. To illustrate this point, we generalize the expression for the cost given in Eq. (1) that now becomes the sum of inverse of the diagonal elements of $\mathbf{C}^{\prime}$ and of $\mathbf{C}_{\mathbf{b b}}^{\prime}$

$$
\begin{aligned}
\operatorname{cost}\left[\mathbf{C}_{\boldsymbol{\alpha} \boldsymbol{\alpha}}^{\prime}, \mathbf{C}_{\mathbf{b b}}^{\prime}\right] & =\operatorname{cost}\left[\mathbf{C}_{\boldsymbol{\alpha} \boldsymbol{\alpha}}^{\prime}\right]+\operatorname{cost}\left[\mathbf{C}_{\mathbf{b b}}^{\prime}\right] \\
& =\sum_{\alpha_{i}} \frac{\lambda_{\alpha_{i}}}{C_{\alpha_{i} \alpha_{i}}^{\prime}}+\sum_{b_{i}} \frac{\lambda_{b_{i}}}{C_{b_{i} b_{i}}^{\prime}}
\end{aligned}
$$

where $\lambda_{\alpha_{i}}$ 's are cost weights of differential data uncertainties and $\lambda_{b_{i}}$ those of IBEs uncertainties. One can see that if the cost associated with IBEs is much larger than 
that of differential data, that is, if $\lambda_{\alpha_{i}} \ll \lambda_{b_{i}}$, the IS/UQ method reverts back to the original formula in Eq. (1).

The sensitivity matrix $\mathbf{S}$ consists of the sensitivity matrix of IBEs and those of applications, that is, $\mathbf{S} \equiv$ $\left\{\mathbf{S}_{\mathbf{b b}}, \mathbf{S}_{\mathbf{a a}}\right\}$, in a notation completely analogous to the definition of the $\mathbf{C}_{\mathbf{m m}}$ in Eq. (12). With this efficient notation, the IS/UQ method retains the efficiency of the formal expressions given in the TSURFER Manual, where IBEs and applications are treated on the same footing, the main difference between the two being that responses of IBEs have been measured experimentally while those of applications have not. The constraint on the target uncertainty remains the same as in Eq. (6).

The $\mathbf{C}_{\mathbf{b b}}^{\prime}$ can be written in terms of the covariance matrix of underlying parameters, for example IBE's solution concentrations, which may affect more than one IBE

$$
\mathbf{C}_{\mathrm{bb}}^{\prime}=\mathbf{S}_{\mathrm{bp}} \mathbf{C}_{\mathrm{pp}}^{\prime} \mathbf{S}_{\mathrm{bp}}^{\mathrm{T}}
$$

where its matrix elements, $C_{p_{i} p_{i^{\prime}}}^{\prime}=\eta_{p_{i}} C_{p_{i} p_{i^{\prime}}} \eta_{i^{\prime}}$, are expressed in terms of the prior covariance matrix of IBE parameters $\mathbf{C}_{\mathbf{p p}}, \mathbf{S}_{\mathbf{b p}}$ is the sensitivity matrix of IBE w.r.t. IBE's underlying parameters, and $\{\eta\}$ are the parameters to be optimized by the IS/UQ method. The optimized uncertainties will guide uncertainty requirements for improved measurements of IBEs. The underlying parameter set $\{p\}$ plays a role similar to the systematic uncertainty described in the previous section.

\section{COST FUNCTION}

According to Eq. (14) a total cost function is a sum of cost function of IBEs and a cost function of differential cross section measurements. It is generally understood that a cost of an IBE, depending on its complexity, is comparable or significantly greater than that of differential cross section measurments. However, we postpone a discussion of IBE cost function until a clearer understanding of its costs has been gained at the National Criticality Experiments Research Center (NCERC) in Nevada where new integral experiments will be designed and performed [19]. Therefore, in this work we consider some of the factors that affect the cost of the differential cross sections measurements alone in order to lay the groundwork for designing a more realistic cost function than the conventional one given by Eq. (1).

It turns out that much of the costs incurred by the experiment are related to the duration of the measurements. Duration of experiments is generally driven by the smallest of the partial cross sections being measured. Since capture cross section is generally the smallest partial cross section, the following discussion pays particular attention to capture cross sections, since we assume that all other cross sections can be measured in parallel during the time it takes to measure capture cross section. Therefore, the following simplified discussion is intended to address the cost of measurements of (all) partial and total cross section measurements, even though the discussion may appear to address mostly measurements of capture cross sections. The following are several factors that affect the length of time needed to collect sufficient data:

CROSS SECTION: Smaller cross sections lead to longer data collection times in order to collect sufficient counts, and the cost of an experiment is proportional to its duration. Of all cross sections, capture cross sections take the longest because they tend to be relatively small. Thus, cross sections other than capture are measured with better statistics, since all cross sections are measured simultaneously. Also, angular distributions of scattering cross sections have issues similar to those of capture due to their small cross sections. This effect can be accounted for in the cost function by defining the cost weights, that is, $\lambda$ 's, to be inversely proportional to the value of the cross section in a given energy group.

NEUTRON FLUX: Lower flux requires a thicker target, and therefore more potentially costly material is needed to get sufficient number of counts. Since neutron flux is facility dependent, it would take into account the fluxes at each facility. However, when all experiments are to be performed at the same facility, its flux affects all measurements equally, and the flux can be thought of as an overall normalization constant. Thicker targets require that self-shielding and multiple scattering be taken into account by the neutron resonance data analysis code SAMMY 2. Doing this may lengthen the data evaluation process and thus moderately increase cost.

TARGET: Its cost (preparation or lease) depends on isotope/elemental natural abundance; namely, its cost is approximately inversely proportional to abundance for stable nuclides. The lease costs are approximately $\$(20$ $\pm 10) \mathrm{K} /($ 1st year $)+\$ 10 \mathrm{~K} /($ each additional year $)$ that includes characterization of the sample for composition, impurities, and uniformity.

OPERATING COST: To illustrate the rates for lowenergy cross sections measured at the RPI or the GELINA, their operating personnel cost rate is $(\$ 600$ $700 /$ hour $) \times(24$ hours/day $) \times(28$ days for an average experiment). In addition to the operating cost, the experimentalist's hourly rate is $(\$ 300 /$ hour $) \times(8$ hours/day $)$ $\times$ (28 days). The overall duration of the experimental data collection is about 4 weeks if everything goes well.

DATA REDUCTION: One additional week for data reduction incurring cost at the same rate, in order to extract cross sections from the raw data. This overhead is incurred regardless of the material/isotope.

DATA EVALUATION: One evaluator may take several months to complete at a cost rate of approximately $\$ 300 /$ hour. When development of a new detector resolution function is required to analyze data, performing Monte-Carlo simulations of that detector, and parameterizing detector's resolution function into a form that can be used in e.g. SAMMY 2, may add few weeks to the data evaluation task.

Low-energy neutron cross sections are typically mea- 
sured for thin and thick targets in two separate runs: thin targets help resolve wide resonances, while thick targets help identify narrow resonances. On the other hand, high-energy neutron cross sections are measured at, for example, LANSCE facility, where a higher repetition rate is possible due to higher energy neutrons. However, its higher neutron flux is offset by a smaller cross section in the high-energy region.

If higher accuracy is needed, rather than trying to decrease the statistical error any further by running experiments in excess of 4 weeks, it is more efficient to decrease systematic errors of background and normalization. This is especially true for capture cross sections whose uncertainty is nearly completely due to systematic uncertainties, like background subtraction, normalization, efficiency of detectors, uncertainty in the flux, etc. For total cross section, it is possible to lower the contributions of systematic uncertainties to the level of statistical uncertainties. Partial cross sections generally have systematic uncertainties (much) greater than statistical uncertainties.

Here we mention the techniques for accurate measurements of experimental background and normalization.

BACKGROUND is measured using the black resonances as filters because they absorb nearly all neutrons. From these measurements one obtains accurate energydependent background to be subtracted from the data. These background measurements will lengthen the time needed to complete the experiment and increase the cost.

NORMALIZATION is determined by measuring a well-known cross section of gold at the $5 \mathrm{eV}$ resonance, or of iron at the $1.1 \mathrm{keV}$ resonance, and a comparison to SAMMY fit of these resonances determines the normalization.

\section{SIMPLE ANALYTICAL EXAMPLE}

We use a simple analytically solvable example of the IS/UQ method to show the benefit of incorporating IBEs into the IS/UQ method. The analytical solutions show that the inclusion of the IBE leads to less stringent data uncertainty needed to achieve the application's response target uncertainty. Let's suppose that in an extremely simple IS/UQ example there is 1) one data point along with its variance, 2) one IBE and its variance, and 3) one nuclear application with a single response uncertainty as the constraint.
This simple problem has an analytical solution. To simplify the analytical solution, we 1) set the initial covariance $\mathrm{C}=1$, where $\mathrm{C}$ is now a number, not a matrix, 2) set all sensitivities $S$ to 1 , that is, $S=1$, where $S$ is now a number, not a matrix, 3) set the $\mathbf{F}_{\frac{m}{k}} \mathbf{C}_{\mathbf{m m}} \mathbf{F} \frac{m}{k}=1$ (a number) in the definition of $\mathbf{C}^{\prime \prime}$ in Eq. (2), and 4 ) set the desired response variance on the right-hand side of the constraint in Eq. (6) to an arbitrary positive number $\delta^{2}$, that is, $\operatorname{var}[R]=\delta^{2}$. Although this simple IS/UQ problem could be solved analytically for arbitrary values of $C$ and $S$ (scalars), the above choice of values is sufficient to illustrate the effect of incorporating IBE into the IS/UQ method.

Without an IBE the optimization amounts to $S C^{\prime} S=$ $x C x=x^{2} \leq \delta^{2}$, since $S=C=1$. The cost is at its minimum for $x^{2}=\delta^{2}$, and $C^{\prime}=x^{2}=\delta^{2}$ is the optimized solution. On the other hand, with an IBE the constraint in Eq. (5) becomes

$$
\begin{aligned}
S C^{\prime \prime} S & =C^{\prime \prime}=C^{\prime}-C^{\prime}\left[1 /\left(C^{\prime}+1\right)\right] C^{\prime} \\
& =x^{2}-x^{2}\left[1 /\left(x^{2}+1\right)\right] x^{2} \leq \delta^{2} .
\end{aligned}
$$

Multiplying both sides of this inequality by $x^{2}+1$ we get $x^{2}\left(x^{2}+1\right)-x^{4} \leq \delta^{2} x^{2}+\delta^{2}$ and solving it we get $x^{2} \leq \delta^{2} /\left(1-\delta^{2}\right)$. The minimum cost is obtained for $C^{\prime}=x^{2}=\delta^{2} /\left(1-\delta^{2}\right)$ which is greater than the $x^{2}=\delta^{2}$ obtained without an IBE, thus demonstrating that the cost is lowered by using IBEs in the IS/UQ method.

\section{CONCLUSIONS}

We have formally extended application of the IS/UQ to systematic and statistical uncertainties. We have shown how the IS/UQ method could be generalized to simultaneously optimize uncertainties of differential data and IBEs data. We presented a simple analytical case that demonstrates advantage of IBEs in the IS/UQ. Future efforts may include introduction of complexity reduction techniques into the IS/UQ method to enable application of the IS/UQ method to complex nuclear applications or for reaching target uncertainty of many and/or complex response [18, 19].

Acknowledgments: We thank Dr. Klaus Guber for useful discussions of differential data measurement costs in Sec. V. This work has been performed with support of the US DOE Nuclear Criticality Safety Program.
[1] M.E. Dunn and N.M. Greene, "AMPX-2000: A CrossSection Processing System for Generating Nuclear Data for Criticality Safety Applications," Trans. Am. NuCL. Soc. 86, 118-119 ( 2002).

[2] N. M. Larson, "Updated Users' Guide for SAMMY: Multilevel R-matrix Fits to Neutron Data Using Bayes' Equations," Report ORNL/TM-9179/7, Oak Ridge National
Laboratory (2006).

[3] M.B. Chadwick et al., "ENDF/B-VII.1 Nuclear Data for Science and Technology: Cross Sections, Covariances, Fission Product Yields and Decay Data," Nucl. DATA SHEETS 112, 2887 (2011).

[4] Oak Ridge National Laboratory, "SCALE: A Comprehensive Modeling and Simulation Suite for Nuclear Safety 
Analysis and Design," Report ORNL/TM-2005/39, Version 6.1 (2011). Available from Radiation Safety Information Computational Center at Oak Ridge National Laboratory as CCC-785.

[5] K. Guber, private communications (2013, 2014).

[6] L.M. Usachev and Yu.G. Bobkov, "Planning an Optimum Set of Microscopic Experiments and Evaluations to Obtain a Given Accuracy in Reactor Parameter Calculations," (English translation of a Russian original), International Atomic Energy Agency Report INDC (CCP)-19/U, January (1972), https://wwwnds.iaea.org/publications/indc/indc-ccp-0019/; IAEA Publication, Conf. 730302, Vol. 1, p. 129 (1973).

[7] J.H. Marable, "The Nutcracker Module of the FORSS System"; C.R. Weisbin et al., "Application of the FORSS Sensitivity and Uncertainty Methodology to Fast Reactor Benchmark Analysis," Report ORNL/TM-5563 (1976), and references therein.

[8] G. Aliberti et al., "Impact of Nuclear Data Uncertainties on Transmutation of Actinides in Accelerator-Driven Assemblies," NuCl. SCI. ENG. 146, 13 (2004).

[9] G. Palmiotti, M. Salvatores, and G. Aliberti, "Methods in Use for Sensitivity Analysis, Uncertainty Evaluation, and Target Accuracy Assessment", Idaho National Laboratory Report INL/CON-07-13356 (2007).

[10] G. Aliberti et al., "Nuclear data sensitivity, uncertainty and target accuracy assessment for future nuclear systems," Ann. Nucl. Energy 33, 700 (2006).
[11] G. Palmiotti et al., "Nuclear Data Target Accuracies for Generation-IV Systems Based on the Use of New Covariance Data," J. Korean Phys. Soc. 59, 1264 (2011).

[12] B. A. Khuwaileh et al., "The Effect of Implicit SelfShielding on the Inverse Sensitivity/Uncertainty Method for Thermal Reactors," Trans. Am. Nucl. Soc. 109, 804 (2013).

[13] G. Arbanas et al., "Integral Benchmark Experiments in the Inverse Sensitivity/Uncertainty Computations," Trans. Am. Nucl. Soc. 109, 808 (2013).

[14] B.L. Broadhead, "Uncertainty Analysis Methods for S/U Criticality Validation Techniques," Trans. Am. Nucl. Soc. 83, 95 (2000).

[15] B.L. Broadhead and B.T. Rearden, "Foundations For Sensitivity-Based Criticality Validation Techniques," Trans. Am. Nucl. Soc. 83, 93 (2000).

[16] M.L. Williams and B.T. Rearden, "SCALE-6 Sensitivity/Uncertainty Methods and Covariance Data," NuCL. Data Sheets 109, 2796 (2008).

[17] C. Wang et al., "Alternative Approach for Importance Ranking of Nuclear Data", ANS Annual Meeting 2014.

[18] B.A. Khuwaileh and H.S. Abdel-Khalik, "Subspace Based Inverse Uncertainty Quantification for Nuclear Data Assessment," Nucl. Data Sheets 123, 57 (2015).

[19] United States Department of Energy Nuclear Criticality Safety Program, "The Mission and Vision of the United States Department of Energy Nuclear Criticality Safety Program for the Fiscal Years 2014-2023", http://ncsp.llnl.gov/NCSP_MISSION_VISION_FY1423.pdf (2014). 